\title{
'So what about the stories?' An exploratory study of the definition, use, and function of Narrative Child Sexual Exploitation Material
}

\author{
Rebecca L. Crookes, Hannah L. Merdian \& Charlotte L. Hassett \\ University of Lincoln, UK
}

Psychology, Crime \& Law, 23:2, 171-179, DOI: 10.1080/1068316X.2016.1239099

\begin{abstract}
The legal and psychological research surrounding online Child Sexual Exploitation Material (CSEM) is focused on visual depictions of children, either as still images or movies. Narrative CSEM (N-CSEM) describes an under-researched area, resulting from difficulties surrounding its conceptualisation, both legally as well as concerning the function for its users. The current study describes an initial attempt in defining N-CSEM in comparison to visual material, based on interviews with users of CSEM and N-CSEM and professionals working with this user group. Thematic Analysis resulted in three super-ordinate themes. All themes were analysed and enriched from the perspectives of user- and service representatives. The study provides insight into N-CSEM as a separate entity from visual CSEM, challenging and informing legal decision-making and assessment and treatment providers for users of CSEM.
\end{abstract}

KEYWORDS: Child sexual exploitation material; Narrative Child Sexual Exploitation Material; thematic analysis; child abusive images; online sex 


\section{'So what about the stories?' An exploratory study of the definition, use, and function of Narrative Child Sexual Exploitation Material}

Approximately one-third of sex offences are now internet-based, though it is impossible to accurately measure the number of people accessing online Child Sexual Exploitation Material (CSEM; Quayle \& Taylor, 2005). Accessing CSEM may potentially be more normative than previously assumed; Ray, Kimonis, and Seto (2014) found that in their community sample of adult male pornography users, $21 \%$ had accessed indecent images of children, and Gannon and O'Connor (2011) reported that 57\% of their sample of community males did not reject an interest in child sexual abuse. However, the primary focus of the CSEM-related research to date remains on visual material (Babchishin, Hanson, \& VanZuylen, 2015), neglecting non-visual material such as Narrative CSEM (N-CSEM). Legal definitions of CSEM differ between countries (Europol, 2005), with variations in the type of material constituting CSEM, its accordance with the legal age of consent, or its classification as sex offending versus censorship offending. The latter point is crucial when discussing the legality of N-CSEM; in the UK, currently N-CSEM is not included in the Sexual Offences Act (2003) but is regulated under the Obscene Publications Act (1959). NCSEM includes written stories or poems, sometimes recorded as audio tapes or depicted as cartoons, describing sexual encounters involving minors (Merdian, 2012). The limited research available has pointed to the potential popularity of N-CSEM amongst CSEM users; Merdian identified that more than half of a sample of convicted CSEM users had possessed narrative material, and conceptualised its appeal based on its easier access and availability in comparison to visual material and the difficulties in its legal classification. To identify the legal and conceptual definition of N-CSEM, Merdian and Hogue (2013) investigated knowledge and attitudes towards N-CSEM and its usage from community samples in the UK, Greece, and Germany. The majority of participants classified sexually explicit narratives 
involving minors as CSEM, referred to their use and engagement as sex offending, and defined the individual engaging in N-CSEM as a sex offender. Nevertheless, participants rated N-CSEM use at the lower end of severity in comparison to sex offences with direct victim contact, spanning a severity continuum depending on three variables: (1) an individual's active involvement (i.e. producing NCSEM) as opposed to passive involvement (i.e. reading N-CSEM); (2) presence of a real, identifiable victim; and (3) age of the involved victim, with lower ages indicating higher severity. However, little is known about the psychological significance of N-CSEM use, its function, and link to a sexual interest in children.

Studies on CSEM have targeted the users of this material as research participants but have not included them as 'service user consultants', despite many men reporting a high level of insight into their own offending processes (Taylor \& Quayle, 2003). Not only may advancement in the legal and psychological definition of N-CSEM have potentially significant implications for its users, but their perspective is vital in the understanding of an under-researched psychological phenomenon. The current exploratory study thus aimed to investigate the definition, use, and function of N-CSEM, based on the experience of users of CSEM and N-CSEM and professionals working in relevant domains. The broader aim of the study was to identify the extent and severity of N-CSEM usage, and to raise the issue of NCSEM as a topic of forensic salience for policy-makers, policing forces, and assessment and treatment providers, as well to provide a starting point for further investigation into this under-researched area.

\section{Methodology}

\section{Study design and recruitment procedures}

Semi-structured interviews were conducted with (1) experts who work in a professional capacity with CSEM users (henceforth termed professional participants; PPs) 
and (2) detected users of visual CSEM (henceforth termed user participants; UPs). PPs were recruited via email using professional networks. UPs were self-selected and recruited through one Community Treatment Center for CSEM users or National Probation Services. UPs were eligible for this study if they were aged over 18 and male (given that the research to date has predominately focused on adult male CSEM offenders); and were either pre-, post-, or in the process of receiving treatment following their offending behaviour.

\section{Participants}

PPs. Eleven participants, aged between 25 and 62 years $(M=42.1$ years; SD = 11.5), were recruited. All participants either had experience of working with CSEM users or had expertise within this field. A range of occupations were represented, including: Sex Offender Treatment Programme facilitators $(n=4)$; psychologists $(n=3)$, a trainee psychologist $(n=$ 1); law lecturers $(\mathrm{n}=2)$; and a Detective Constable with the police $(\mathrm{n}=1)$.

UPs. Eleven males volunteered for the study. Participants ages ranged from 28 to 66 years $(M=46$ years; $S D=14.88)$. To ensure anonymity, previous and current occupations of the UPs are not disclosed. In terms of marital status, $n=6$ were single, $n=2$ were married, $n$ $=1$ was engaged, $\mathrm{n}=1$ was divorced, and $\mathrm{n}=1$ was widowed. Six participants had children. All UPs had been arrested or convicted for downloading or possession of indecent material (CSEM), and one also being convicted of distributing CSEM. In terms of sentencing, one participant received a custodial sentence, one received a suspended sentence, and nine participants reported receiving either a probation or community order.

It is important to note that, while unlikely, some participants may not have directly encountered N-CSEM as research participation did not require a disclosure of their behaviour; however, all participants volunteered with full knowledge of the research question and had been exposed to the topic through, for example, group discussions, the literature, or supervision. 


\section{Study procedure and analysis}

For PPs, a Delphi-method approach was employed, involving a feedback process of the initial data analysis to participants for further commenting, a commonly used method to achieve a reliable agreement of a group of experts (Geist, 2010). Interviews with PPs (faceto-face or via telephone/online real-time communication tools) were conducted using a semistructured interview protocol, focusing on definitions of CSEM and N-CSEM, the legal categorisation of N-CSEM, whether N-CSEM was understood to be a precursor to contact sex offending, and on the assessment and treatment of N-CSEM users.

Interviews lasted between 10 and 40 minutes. Following the initial analysis of the data and according to the Delphi-method protocol, an interim report was emailed to the participants for further commenting. For UPs, no feedback process was involved to protect their confidentiality and anonymity. A user-focused, semi-structured interview protocol was employed, focusing on an understanding of the participants' general offending behaviour of CSEM, N-CSEM usage in specific, their motivations for the offending behaviour; and how their material was perceived to be related to sexual arousal and fantasy. Interviews with UPs lasted approximately 30-40 minutes.

The study had ethical approval in accordance with the British Psychological Society Code of Ethics and Conduct (2009). Interviews from both stages of the study were digitally recorded and transcribed verbatim. Interview data were analysed using Thematic Analysis (Braun \& Clarke, 2006).

\section{Results and discussion}

Based on interviews with PPs, three super-ordinate themes emerged: (1) conceptual and legal definitions of N-CSEM; (2) considering harm of N-CSEM: relationship to contact sex offending and impact on society; and (3) function of N-CSEM. Interviews with UPs supported and enriched the identified themes. 


\section{Conceptual and legal definitions of N-CSEM}

There was some difficulty amongst PPs to provide a consensus definition of N-

CSEM; collectively, PPs considered N-CSEM to be stories, diaries, cartoons, and other textbased material that depicts children in a sexual context. For example, PP11 reported that they had worked with individuals who had ' ... diaries where the defendant had written down what he wanted to do to certain children from a sexual point of view, and to me, that's narrative'. All PPs classified N-CSEM as CSEM and as material that is used to achieve sexual arousal. However, PP4 and PP10 cautioned that a legal definition of NCSEM should be considered carefully, due to its blurred position between obscenity and art. The concept of Freedom of Speech was often referred to; PP10 compared the regulation of N-CSEM to that of hate speech: 'I think if you're going to control this kind of material...you've either got to say that we have got evidence that it does lead to harm (for example, that the material is used for grooming children for sexual purposes), or you've got to put it in the category of 'hate speech', and so you draw the link with the racist speech and say we control it because of its nature, not necessarily because we can always show that racist speech can lead on to other racist action, but simply because we regard it unacceptable speech in our society.'

PPs further listed the lack of an identifiable victim and the lack of measurable harm to a child as arguments against the criminalisation of N-CSEM. This point was supported by UPs, who clearly distanced N-CSEM from visual CSEM based on the lack of an identifiable victim: 'But I considered that the stories were not as bad, as it was a story, no one is actually being physically abused so it's just out of somebody's fantasy or imagination' (UP3). However, UP5 drew parallels from visual CSEM to N-CSEM: 'People can have distorted thinking around imagery, that there's no victim in imagery or that the images aren't real, which is wrong'. Further, UP5 thought that both N-CSEM and visual CSEM caused equal harm: 'Ultimately, children are either being harmed or are at risk'. Overall, PPs agreed that 
more research is required to understand whether the material should be considered harmful; PP4 and PP10 suggested including the purpose of sexual gratification in the definition of $\mathrm{N}$ CSEM, and to focus on the intent of producing and using the material rather than its content. Other PPs argued that N-CSEM could depict a contact child sexual abuse that has already happened, or the material could encourage users to engage in similar behaviour. All PPs considered users, creators, and distributors of N-CSEM as sex offenders, however, acknowledged difficulties surrounding the legal application of the label.

The majority of UPs thought that N-CSEM should not be illegal: 'The problem is punishing people for reading is a very slippery slope... I don't think it really wants to go down criminalising every form of sexuality' (UP4), and were uncertain about the standards of prohibiting N-CSEM: 'If you've got an image of a naked child, it's a naked child. With a story you don’t know' (UP3). A comparison was also drawn to stories describing violence: 'There's a lot of films that are about murder but you know, and there's a lot of books about murder but not everybody is going to go out and murder, are they?' (UP11). Overall, the discussion about the conceptual and legal definition of N-CSEM was closely linked to the perceived harm of N-CSEM, and its function for the individual user.

\section{Considering harm of N-CSEM: relationship to contact sex offending and impact on society}

Related to above, many participants discussed the issue of the harm caused by $\mathrm{N}$ CSEM, both with regard to the relationship between N-CSEM and contact sex offending; and aspects of harm relating to societal morals.

Relationship to contact sex offending. Overall, PPs agreed that in their experience, users of CSEM do not progress to contact offending; however, acknowledged the importance of individual factors. Some PPs compared the risk potential of N-CSEM to visual CSEM: ' $[\mathrm{N}-\mathrm{CSEM}]$ could be a precursor to [CSEM], which could then potentially lead to contact 
offending, as an interest in children must lend itself to curiosity and an itch that, therefore, needs to be scratched' (PP11). Some PPs stated that N-CSEM could be more harmful than CSEM, as a stronger reinforcer of 'cognitive distortions' (PP2 and PP4) or as a 'fantasy generator' (PP7), which was confirmed by UPs. However, other PPs reflected on the lack of empirical data supporting a link between visual CSEM and contact sex offending, that may also apply to N-CSEM: '[They] have a barrier that prevents them from going on to contact offend' (PP6). On this point, the UPs provided some interesting reflections on the risk related to the level of engagement with N-CSEM, confirming to the active-passive severity continuum identified above: 'I think it's more severe to write it because you're putting the material out there, you've got something inside of you that you're wanting to put down on to paper, electronically, and actually share with other people' (UP10).

Similar to the PPs, there was no consensus amongst UPs concerning the risk level posed by N-CSEM in comparison to visual CSEM: some participants believed that CSEM was more severe than N-CSEM given the harm being caused to an identifiable child. However, UPs acknowledged that N-CSEM may encourage or lead to other offending behaviour, including contact offending. Some participants believed that N-CSEM was more severe in terms of its link to a potential contact offence: 'Because I think that a person who would use a narrative, who's painting a mental picture would continue to want that mental picture to go further and I think at that point, erm, the narrative is stronger than the image to take people to the next level.' (UP8)

Impact on society. Besides the direct link to the sexual abuse of a child, participants also discussed a broader definition of harm N-CSEM may pose to society. Some PPs said that consumers (i.e. users, creators and/or distributors) of N-CSEM 'encourage and fuel the child abuse business' (PP11), in a 'supply and demand' (PP5) model. PP6 recalled a case where an offender (classified as high risk) ' ...had only been convicted of internet offences, but had a 
range of narrative material as well; including a manual called "Boy Love" - an instruction manual for abusing boys that he distributed to others'. PP9 explained that '[the internet] legitimises sexual interest in children by creating networks of like-minded people and making materials more accessible. Therefore, distributing materials normalises this behaviour, which cannot be good for society'. PP11 was also concerned that this material would be impressionable on younger people who are exploring their sexual interests: 'If they have access to [NCSEM] material that describes and depicts child sexual abuse, they could potentially get ideas from reading this material about contact offending'. However, PPs questioned the theoretical and empirical basis of a social learning effect; PP3 and PP4 made reference to the lack of an empirical link between violent material on the commission of violent offending. Additionally, the PPs acknowledged that they had only encountered NCSEM in conjunction with other offending behaviour, which may confound the perceived risk related to N-CSEM.

\section{Function of N-CSEM}

The function of N-CSEM refers to the individual purpose of using N-CSEM and its place in the offending cycle (if any), which may inform and interact with the above themes. PPs agreed that N-CSEM may be a fantasy-enabler, with several PPs stating that fantasies could be enhanced even more with narrative as opposed to visual CSEM. For example, PP5 stated: 'They are able to easily place themselves in the scenario, they can choose the age, gender and appearance of the child and what happens in the scenario' and PP8 added: '[NCSEM can] portray the children as enjoying the encounters and as willing [for the user]'. On the other hand, PP9 stated that N-CSEM may have the same function as visual CSEM, however, N-CSEM may be an 'acceptable', potentially legal substitute to visual CSEM while still facilitating sexual interest in children. Similarly, UPs focused on sexual fantasies as a potential mediator between the material and potentially subsequent offending behaviour: 'I 
suppose in a way they created the fantasy then, they can create an image of the person, they can create a situation, they can create the atmosphere, the surroundings, they can create everything about it in their own head and write it down, and share their fantasy with someone else.' (UP11)

There appeared to be consensus that N-CSEM may be specifically appealing for a particular type of person: 'I know some people don't have a great imagination or are unable to visualise very well' (UP9). Similarly, some participants reported that 'increased concentration' (UP8) was needed when using N-CSEM compared to visual CSEM: 'It takes a while I'm sure to read a story and that sort of thing, so unless they're building time and that sort of thing, it's [that] the images are a quicker way [of] (...) instant release' (UP11). Thus, it appeared that N-CSEM was perceived to be functionally similar to CSEM, but directed at different target audiences.

\section{Conclusion}

This study aimed to explore the issue of narrative CSEM based on the experiences of users of CSEM and professionals with relevant expertise. N-CSEM is an under-researched topic and the presented data showed the need for further research to clarify the legality and potential risk of N-CSEM usage, and to inform assessment and treatment approaches for CSEM users.

The study findings fall broadly into two domains: the conceptual issues surrounding $\mathrm{N}$-CSEM (relating to its definition and perceived harmfulness); and operational issues (functions of engagement with N-CSEM). An interesting debate emerged concerning the definition of N-CSEM; overall, PPs agreed that N-CSEM should be perceived as a form of CSEM and should be illegal if its purpose is to achieve sexual gratification. But both professionals' and UPs' responses reflected the difficulties concerning a legal classification of N-CSEM, in terms of defining obscenity versus freedom of expression and free speech, 
differing levels of explicitness, the lack of an identifiable victim, and the comparison to other, legal descriptions of illegal actions (such as murder stories). In summary, the following working definition of N-CSEM is suggested to reflect the participants' responses: Narrative CSEM refers to stories, diaries, cartoons, and other text-related material that depicts a child under the age of 18 years in a sexual context, describing sexual activities and used for sexual gratification by the recipient. However, we do not perceive this definition as final but as a starting point of engagement with this topic, and invite readers to critically engage with this working definition, and for future research to challenge and refine this initial classification.

The participants' responses also communicated that criminalisation of N-CSEM must be approached with caution, given the lack of clarity concerning the harm this material presents. Drawing on the research context on visual CSEM, few users appear to escalate in their offending behaviours from CSEM use to contact sex offending (Seto, Hanson, \& Babchishin, 2011), and the ones who do appear to be subgroup of CSEM users characterised by access to children, high levels of anti-sociality, and few psychological barriers towards acting on their sexual interest in children (Babchishin et al., 2015). As with visual CSEM, participants stated that the risk of using N-CSEM appears to be moderated by individual factors, relating to the function of one's N-CSEM use. This resonates with the discussion surrounding the negative effects of exposure to violent media (Anderson, Carnagey, \& Eubanks, 2003) or exposure to pornography at an early age (e.g. Horvath et al., 2013). Central to this argument is the function of N-CSEM use, relating to the needs a user intends to meet with the material. Taylor and Quayle (2003) originally identified six functions of CSEM use, namely, sexual arousal, collection purposes, to foster social contacts, to escape real life problems, and as a 'safe' place to explore their sexual preferences without acting on them. Other research has expanded on the original functions and offence motivations of CSEM users (e.g. Merdian, Curtis, Thakker, Wilson, \& Boer, 2013; Sheldon \& Howitt, 2007; 
Surjadi, Bullens, Van Horn, \& Bogaerts, 2010). Despite the identified similarities in the function of N-CSEM and visual CSEM in this research, both PPs and UPs pointed to the potentially more stimulating nature of N-CSEM as fantasy enhancers. The debate surrounding access to N-CSEM points to CSEM offending as a context-enabled behaviour which can be controlled both through situational interventions (e.g. Wortley \& Smallbone, 2006) as well as through enhancing internal inhibitions (e.g. Babchishin et al., 2015). We hoped for this study to become a starting point in researching N-CSEM and in raising awareness of non-visual material in online sexual offending. Overall, the study pointed to a strong individual element in the potential risk related to N-CSEM use, and the role of internal inhibitions in the engagement process. This topic defines an under-researched area; it is yet to investigate what impact and role N-CSEM has within the offending cycle, before recommendations can be made to policing, law-makers, and sex offender assessment and treatment providers. 


\section{References}

Anderson, C. A., Carnagey, N. L., \& Eubanks, J. (2003). Exposure to violent media: The effects of songs with violent lyrics on aggressive thoughts and feelings. Journal of Personality and Social Psychology, 84(5), 960-971. doi:10.1037/0022-3514.84.5.960

Babchishin, K. M., Hanson, R. K., \& VanZuylen, H. (2015). Online child pornography offenders are different: A meta-analysis of the characteristics of online and offline sex offenders against children. Archives of Sexual Behavior, 44(45), 45-66. doi:10.1007/s10508-014-0270-x

Braun, V., \& Clarke, V. (2006). Using thematic analysis in psychology. Qualitative Analysis in Psychology, 3(2), 77-101. doi:10.1007/1478088706qp063oa

British Psychological Society. (2009). Code of ethics and conduct. Retrieved from http://www.bps.org.uk/system/files/documents/code_of_ethics_and_conduct.pdf

Europol. (2005). Europol public opinion: Child pornography legislation within the European Union. Retrieved from http://www.internationalresourcecentre.org/en_X2/Documents/LegislationEuropol_20 05.pdf

Gannon, T., \& O'Connor, A. (2011). The development of the interest in child molestation scale. Sexual Abuse: A Journal of Research and Treatment, 23(4), 474-493. doi:10.1177/1079063211412390

Geist, M. R. (2010). Using the Delphi method to engage stakeholders: A comparison of two studies. Evaluation and Program Planning, 33, 147-154. doi:10.1016/j.evalprogplan.2009.06.006

Horvath, M. A. H., Alys, L., Massey, K., Pina, A., Scally, M., \& Adler, J. R. (2013). Basically...porn is everywhere: A rapid evidence assessment on the effects that 
access and exposure to pornography has on children and young people. Project Report. London: Office of the Children's Commissioner for England.

Merdian, H. (2012). Offenders who use child sexual exploitation material: Development of an integrated model for their classification, assessment and treatment (doctoral thesis). University of Waikato, Hamilton.

Merdian, H., \& Hogue, T. E. (2013). Exploring community attitudes regarding narrative child pornography. Report prepared for the College Research Fund, University of Lincoln, Lincoln.

Merdian, H. L., Curtis, C., Thakker, J., Wilson, N., \& Boer, D. P. (2013). The three dimensions of online child pornography offending. Journal of Sexual Aggression: An International Interdisciplinary Forum for Research, Theory and Practice, 19(1), 121132. doi:10.1080/13552600.2011.611898

Obscene Publications Act. (1959). (c.66). London: The Stationary Office.

Quayle, E., \& Taylor, M. (2005). Viewing child pornography on the Internet: Understanding the offence, managing the offender, helping the victims. Dorset, UK: Russell House Publishing Ltd.

Ray, J. V., Kimonis, E. R., \& Seto, M. C. (2014). Correlates and moderators of child pornography consumption in a community sample. Sexual Abuse: A Journal of Research and Treatment, 26(6), 523-545. doi:10.1177/1079063213502678

Seto, M. C., Hanson, R. K., \& Babchishin, K. M. (2011). Contact sexual offending by men arrested for child pornography offences. Sexual Abuse: A Journal of Research and Treatment, 23(1), 124-154. doi:10.1177/1079063210369013

Sexual Offences Act. (2003). (c42). London: Stationary Office.

Sheldon, K., \& Howitt, D. (2007). Sex offenders and the internet. Chichester: John Wiley \& Sons. 
Surjadi, B., Bullens, R., Van Horn, J., \& Bogaerts, S. (2010). Internet offending: Sexual and non-sexual functions within a Dutch sample. Journal of Sexual Aggression: An International Interdisciplinary Forum for Research, Theory and Practice, 16(1), 4758. doi:10.1080/13552600903470054

Taylor, M., \& Quayle, E. (2003). Child pornography: An internet crime. East Sussex: Brunner-Routledge.

Wortley, R., \& Smallbone, S. (2006, May). Child pornography on the internet. Problemoriented guides for police, problem-specific guides series 41. Washington, DC: US Department of Justice, Office of Community Oriented Policing Services. 